\title{
Traditional Uses and Sustainable Collection of Ethnobotanicals by Aboriginal Communities of the Achanakmaar Amarkantak Biosphere Reserve of India
}

\author{
Tarun Kumar Thakur ${ }^{1, ~ *, ~ Y o g e s h ~ K u m a r ', ~ A r v i n d ~ B i j a l w a n ², ~ M a n m o h a n ~ J ~ D o b r i y a l ~}{ }^{3}$ \\ ${ }^{1}$ Department of environmental Science, IG National Tribal University, Amarkantak, India \\ ${ }^{2}$ Indian Institute of Forest Management (IIFM), Bhopal, India \\ ${ }^{3}$ College of Forestry (ACHF), Navsari Agricultural University, Navsari, India
}

Email address:

tarun_2711@yahoo.co.in (T. K. Thakur)

*Corresponding author

\section{To cite this article:}

Tarun Kumar Thakur, Yogesh Kumar, Arvind Bijalwan, Manmohan J Dobriyal. Traditional Uses and Sustainable Collection of Ethnobotanicals by Aboriginal Communities of the Achanakmaar Amarkantak Biosphere Reserve of India. Frontiers in Environmental Microbiology. Vol. 3, No. 3, 2017, pp. 39-49. doi: 10.11648/j.fem.20170303.11

Received: February 27, 2017; Accepted: March 9, 2017; Published: June 16, 2017

\begin{abstract}
In the due course of study, focus was prearranged on the traditional use of trees, herbs and shrubs (ethnobotanicals) which are little or unknown to modern societies. Through questionnaire and interviews, the present study was attempted to collect the information about the people who still live in traditional world. A total 40 tree species, 94 herbs including tubers, grasses, climbers and 13 shrubs utilize by aboriginal communities of Achanakmaar Amarkantak Biosphere Reserve (AABR), were renowned/ explored for different utilization pattern. The most important tree species collected from the forests and nearby village areas by the local people are Madhuca indica, Buchanania lanzan, Diospyrus melanoxylon, Mangifera indica, Shorea robusta and Terminalia tomentosa etc. Similarly, herbs collected are Eclipta alba, Panicum antidotale, Smithia conferta, Phyla nodiflora, Dioscorea bulbifera, Curculigo orchioides, Oxalis corniculata, Portulaca oleracea, Echinochloa colona, Solanum nodiflorum, Achyranthus aspera, Leucas aspera, Corchorus trilloularis, Cassia tora etc. The most common reported shrubs of these areas are Phoenix sylvestris, Randia dumetorum, Zizipus zilopyrus and Lantana camara. The reported botanicals have variety of uses like vegetable, fruit, furniture, religious use, rituals use, and for handloom preparation. Besides the consumption value, forest also source of subsistence for this hidden and marginalized society of the world. The current study confirmed that there is a vital necessity for documentation of traditional knowledge associated to the Bagia aboriginals and others insubstantial cultural inheritance regarding traditional plant uses. Further, it can provide a baseline ethnobotanicals utilization pattern data that may be guiding parameter for the prioritization and conservation of these natural resources along with bio-prospecting indigenous traditional knowledge.
\end{abstract}

Keywords: Aboriginals, Forests, Sustainable, Aabr, Ethnobotanicals

\section{Introduction}

The human beings are closely associated with forest for their existence and civilization. The relationship between human being and forest has been important for the development of society (Riter et al. 2013). The development of society is based on various function of forest like productive, ecological, social and cultural etc.
The aboriginals /indigenous people continuously (generation to generation) associated with forest and often possess a broad knowledge base about the complex ecological behavior of forest in their own localities (Gadgil et al. 1993). Most of people live in and around forest belongs to tribal communities. These forest dwellers collect different type of forest produce from forest either for their own consumption or for sale in the market. The collection intensity of forest produces mainly dependent 
on availability, knowledge and easily accessibility of it (Kala, 2009). Millions of people, particularly tribal and rural communities in many developing countries still collect and consumed a wide variety of wild plant resources to meet their food requirements (FAO, 2004; Balemie et al. 2006; Bharucha et al.2010, Dobriyal, et al, 2015). Forest is an important source of highly nutritive value food for tribes. Intensive studies concerning its nutritional role have also been highlighted in many surveys around the world (Tanji et al. 1995; Ogle et al. 2001; Bonet et al. 2002; Guarrera et al. 2003; OgoyeNdegwa et al. 2003; Ertug, 2004; Tardio et al. 2005; Javier et al. 2006). These wild plants provide balance diet by supplementing essential nutrients and minerals for human being. The wild plants have been recognized as potential source of nutrition than conventionally eaten crops (Grivetti et al. 2000). Due to modernization in society (advanced communication, transportation and technology) there is a mixing of culture and customs of these forest dwellers, but still they have endowed with many traditional rituals, beliefs, norms and practices which are highly associated with forest. Tribal communities have strong cultural, spiritual and livelihood link with forest. The Achanakmaar Amarkantak Biosphere reserve (AABR) in Central India is known to home of several Non Timber Forest Produce(NTFPs), rare and endangered medicinal aromatic plant and different type of forest ranging from subtropical to dry deciduous, which greatly contribute to livelihoods of inhabited tribal communities. The inhabited tribes in AABR are mostly belonging to Baiga, Gond, Kol, Kanwar, Pradhan and Panka communities. Every plant on earth has utility but bio-prospecting this resource is a big task. These forest dwellers have vast knowledge about appropriate uses of forest products. They are very well aware that plants play very important role in generating the ecological services and natural resources on which they depend. They consider every part of environment from trees to rivers as very important part of their life as they are the only means of human survival as per their understanding (Pandey, 1997). Forest dependent communities are usually located far from cities and fertile agriculture areas, so these communities fulfill their almost day to day need from forest (Yeo-Chang, 2009). Most of the basic needs of these tribe's like food (leafy vegetables, fruit, and kodo, kutki, sama grains), shelter (Bamboo, grasses and leafs) and income (by selling leaf of bahunia, mushroom and mahua flowers etc.) are fulfilled through forest. Tribal communities have symbiotic relation with forest they (forest and tribe) depend on each others. The tribal communities have extensive knowledge about the traditional uses of these natural resources which are little or unknown to modern societies. The knowledge remained just with the people who are acquainted with these uses and has rarely been documented otherwise (Burgi et al. 2013). There is a real need for field-level research, synthesis and collection of information on tribal-forest association. With this background, a need was felt to collect in-depth information on the tree species growing in AABR and used by tribal groups in view of documenting the knowledge which may be under threat due to the influence of modernization. This paper thus aims to highlight and record in detail the traditional knowledge of tribal groups on the use of various tree species growing in AABR.

\section{Methodology}

\subsection{Study Area}

The AABR lays between $21^{\circ} 15^{\prime}$ to $22^{\circ} 58^{\prime} \mathrm{N}$ latitude and $81^{\circ} 25^{\prime}$ to $82^{\circ} 5^{\prime} \mathrm{E}$ longitude. The Achanakmar Amarkantak Biosphere Reserve is spread over an area of 383551.0 ha in Deccan Peninsula bio-geographic zone of Chhattisgarh and Madhya Pradesh and comprises of tropical moist deciduous to tropical dry deciduous forests. .The total geographical area of AABR is 383551.0 ha of which 55155.0 ha is under the core zone and remaining 28396.889 ha comprises the buffer zone. The zonation in AABR makes clear the ecological and socioeconomical independence between each zone of AABR. The core region of the AABR is situated in Chhattisgarh state whereas the buffer and transition zones lie partly both in Madhya Pradesh and Chhattisgarh. The AABR possessed rich plant and animal diversity. It is similarly known for its cultural diversity, as it is colonized by number of tribal and non-tribal group of people.

\subsection{Survey Methods}

The present study was carried out in buffer zone and core zone's villages of AABR. A total of 7 villages in buffer zone namely Aamadoab, Kewanchi, Piper Khuti, Kachhra tola, Roopan dand, Pattar coni, Madna dipo and 5 villages of core zone namely Lamni, Birar pani, Chhiritta, Atariyaa and Ranchaki were selected for intensive study of traditional knowledge on the use of flora of AABR (Table 1). The location map of study area is depicted in Fig 1. The selected villages of core zone were dominated by tribal communities while buffer zone has both tribal and non tribal communities. The randomly selected tribals hamlets and families from different communities were questionnaire survey in the selected villages of AABR. The important part of the questionnaire were discussed with 60 to 70 years villagers because they have great experience regarding the use of forest products. The females provided the information mainly regarding the wild vegetables. Through questionnaire survey and interviews the information was collected on the indigenous uses of tree species, their processing techniques, consumption pattern, plant part use, local name of tree species and their occurrence in AABR. The questions were also asked on the role of forest products on the economy of tribal community and non tribal communities reside in and around the biosphere reserve. Apart from questionnaire 
survey, the local people were encouraged to give their ideas and perceptions on various uses of herbs, shrubs and tree species. Information was collected on ethnobotanicals by participating in various cultural activities of the local tribal people. Efforts were made to scrutinize the tree species used in various socio-cultural practices of the local people including childbirth, death and marriage ceremony.
The related cultural practices and norms for collection of a variety of forest produce and parts of particular tree species were also documented through group discussion. The experienced local people were also requested to escort during the forest survey for identification of tree species and related indigenous knowledge.

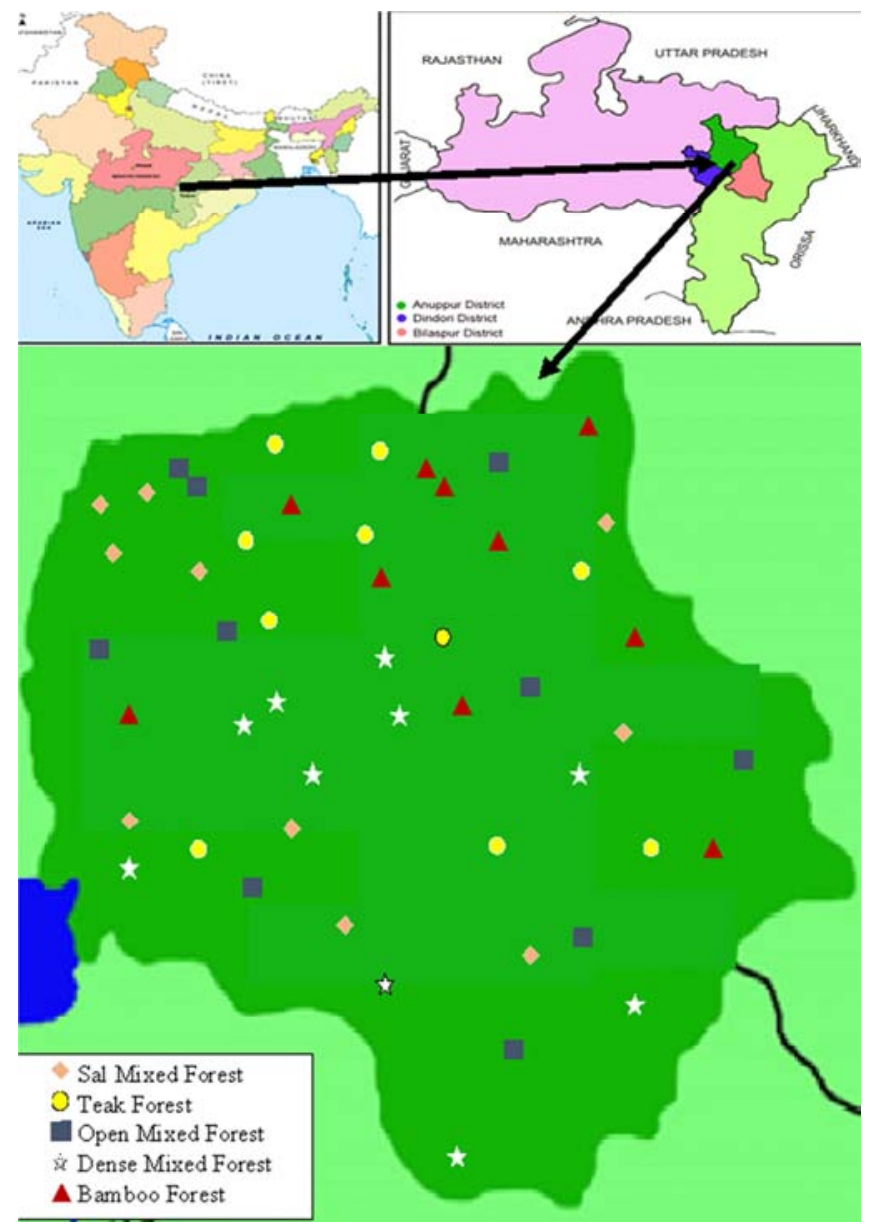

Figure 1. Study area and location of field study of achanakmaar amarkantak biosphere reserve.

Table 1. List of villages, surveyed in aabr.

\begin{tabular}{|c|c|c|c|c|c|c|}
\hline \multicolumn{7}{|c|}{ Core zone } \\
\hline S. $\mathbf{N}$ & Village Name & GPS Location & Elevation MSL (m) & Informants & Localities & Population (2011 census) \\
\hline 1. & Lamni & $\mathrm{N}-22^{0} 32^{\prime} 32.53^{\prime \prime} \mathrm{E}-81^{0} 44^{\prime} 37.20^{\prime \prime}$ & 569 & 18 & 4 & 634 \\
\hline 2. & Birar pani & $\mathrm{N}-22^{0} 32^{\prime} 36.30^{\prime \prime} \mathrm{E}-81^{0} 38^{\prime} 36.70^{\prime \prime}$ & 781 & 13 & 3 & 75 \\
\hline 3. & Chhirhatta & $\mathrm{N}-22^{0} 31^{\prime} 23.50^{\prime \prime} \mathrm{E}-81^{0} 41^{\prime} 46.60^{\prime \prime}$ & 446 & 11 & 3 & 94 \\
\hline 4. & Atariya & $\mathrm{N}-22^{0} 34^{\prime} 08.07^{\prime \prime} \mathrm{E}-81^{0} 45^{\prime} 12.71^{\prime \prime}$ & 598 & 17 & 5 & 668 \\
\hline 5. & Ranchaki & $\mathrm{N}-22^{0} 34^{\prime} .445^{\prime \prime}$ E- $81^{0} 41^{\prime} .553^{\prime \prime}$ & 701 & 15 & 3 & 342 \\
\hline \multicolumn{7}{|c|}{ Buffer Zone } \\
\hline S. N & Village Name & GPS Location & Elevation MSL (m) & Informants & Localities & Population (2011 census) \\
\hline 1. & Aamadob & $\mathrm{N}-22^{0} 37^{\prime} 17.68^{\prime \prime}$ E-81 ${ }^{0} 44^{\prime} 10.77^{\prime \prime}$ & 656 & 21 & 4 & 1208 \\
\hline 2. & Kewanchhi & $\mathrm{N}-22^{0} 37^{\prime} 17.73^{\prime \prime} \mathrm{E}-81^{0} 46^{\prime} 44.82^{\prime \prime}$ & 578 & 23 & 6 & 846 \\
\hline 3. & Pipar Khuti & N-22 ${ }^{0} 39^{\prime} 36.33^{\prime \prime}$ E-81 ${ }^{\circ} 52^{\prime} 08.80^{\prime \prime}$ & 596 & 27 & 8 & 786 \\
\hline 4. & Kachhra tola & N-22 $400^{\prime} 47.45^{\prime \prime}$ E-81 $52^{\circ} 59.82 ”$ & 600 & 11 & 2 & 332 \\
\hline 5. & Roopan dand & $\mathrm{N}-22^{0} 41^{\prime} 52.17^{\prime \prime} \mathrm{E}-81^{0} 53^{\prime} 31.51^{\prime \prime}$ & 591 & 13 & 3 & 1003 \\
\hline 6. & Paterkoni & $\mathrm{N}-22^{0} 42^{\prime} 55.83^{\prime \prime} \mathrm{E}-81^{0} 53^{\prime} 48.02^{\prime \prime}$ & 594 & 09 & 2 & 787 \\
\hline 7. & Madana & $\mathrm{N}-22^{0} 43^{\prime} 42.82^{\prime \prime}$ E- $81^{0} 54^{\prime} 07.54^{\prime \prime}$ & 604 & 23 & 5 & 861 \\
\hline
\end{tabular}

Parts used by local people were cross-checked with other people and evaluate the result from personal interview and discussions in group with local people provide much valuable and specific information regarding the traditional uses of plants. 


\section{Results and Discussion}

The present study brought it in to the light that there are many traditional uses of forest which are well known by indigenous communities meanwhile the modern society have very limited or insufficient knowledge on these uses. In the same way various plants used by the local people of AABR are such type of distinctive uses of the plants. A total 40 tree species, 94 herbs including tubers, grasses, climbers and 10 shrubs utilize by local people of AABR, were renowned during the present study (Table 2, 3 and 4). The most important tree species collected from the forests and nearby village areas by the local people are Madhuca indica, Buchanania lanzan, Diospyrus melanozylon, Mengifera indica, Phyllanthus emblica, Syzygium cumini, Terminalia chebula, Terminalia bellirica, Shorea robustra and Terminalia tomentosa. The local people have multiple uses of ethnobotanical plants, of these 13 uses are recognized during the present study (Figure 2) and The informants revealed that different morphological plant parts were used by local people (Figure 3) and their distribution of recorded ethnobotanical plants into families are depicted in figure 4. The most common herbs collected from forest or nearby village areas by the local people are Eclipta alba, Panicum antidotale, Smithia conferta, Euphorbia heterophylla, Phyla nodiflora, Dioscorea bulbifera, Curculigo orchioides, Oxalis corniculata, Portulaca oleracea, Echinochloa colona, Solanum nodiflorum, Achyranthes aspera, Leucas aspera, Corchorus trilloularis, Cassia tora etc. These herbs mostly used for vegetable and medicinal purpose and some time also used for fruit and grain. The most common reported shrubs of these areas are Phoenix sylvestris, Randiaa dumetorum, Zizipus zilopyrus and Lantana camara. The reported shrubs have variety of uses like vegetable, fruit and for handloom preparation. Most of species are used as food (38\%) and medicine (54\%). Besides, the plant species are used to execute cultural practices $(6.9 \%)$, for fuel wood $(5.5 \%)$, house construction $(6.9 \%)$, making agriculture tools and instruments $(4.1 \%)$, furniture $(3.5 \%)$, as fish poison $(1.3 \%)$, fodder, oil making, liquor preparation, rope, cup and plate making, and bio-fencing. Seed, dye, leaf, tubers, mushrooms, gum, resin, root, fruit, flower and twig of plants are also used and collected from the forests. The gum mainly collected from Shorea robusta, Anogeissus latifolia, Boswellia serrata and Sterculia urens. The species which used for day to day needs like house construction, making agricultural implements, fuel wood, fodder purpose, and manufacturing boundary wall are cut down around the year as per needs. However, there are a few tree species, such as, Ficus religiosa, Aegle marmelos, Syzygium cumini, Madhuca indica and Shorea robusta, having socio-cultural importance to local people are harvested/felled after performing some local rituals or prayer practices.

Table 2. Herbaceous flora of aabr with local name, scientific name and family name along with their uses.

\begin{tabular}{|c|c|c|c|c|c|}
\hline S. No & common name & Scientific name & Family & Habit & uses \\
\hline 1. & Katua shak & Alternanthera philoxeroides (Mart.) Griseb. & Amaranthaceae & $\mathrm{H}$ & $\mathrm{V}$ \\
\hline 2. & Kubbi & Ageratum conyzoides (L.) L. & Asteraceae & $\mathrm{H}$ & M \\
\hline 3. & Kurie & Bidens pilosa $\mathrm{L}$. & Asteraceae & $\mathrm{H}$ & M \\
\hline 4. & Safed murga & Celosia argentea $\mathrm{L}$. & Amaranthaceae & $\mathrm{H}$ & $M \& V$ \\
\hline 5. & Bhrangraj & Eclipta alba (L.) Hassk. & Asteraceae & $\mathrm{H}$ & M \\
\hline 6. & Kutki & Panicum antidotale Retz. & Poaceae & G & $\mathrm{F}$ \\
\hline 7. & Grass lily & Iphigenia indica (L.) A.Gray ex Kunth & Poaceae & G & Fo \\
\hline 9. & Naichi bhaji & Smithia conferta $\mathrm{Sm}$. & Fabaceae & $\mathrm{H}$ & V \\
\hline 10. & Kanghi & Blainvillea acmella (L.) Philipson & Asteraceae & $\mathrm{H}$ & M \\
\hline 11. & Khal muriya & Tridax procumbens (L.) L. & Asteraceae & $\mathrm{H}$ & M \\
\hline 12. & Dudhali & Sopubia delphinifolia G.Don & Scrophulariaceae & $\mathrm{H}$ & M \\
\hline 13. & Akarkara & Spilanthes paniculata Wall. ex DC. & Asteraceae & $\mathrm{H}$ & M \\
\hline 14. & Chauli & Alysicarpus monilifer (L.) DC. & Fabaceae & $\mathrm{H}$ & M \\
\hline 15. & Doodhi & Euphorbia heterophylla DesF. & Euphorbiaceae & $\mathrm{H}$ & M \\
\hline 18. & Sauri & Alysicarpus vaginalis (L.) DC. & Fabaceae & $\mathrm{H}$ & M \\
\hline 19. & Ghughunia & Crotalaria retusa $\mathrm{L}$. & Leguminosae & $\mathrm{H}$ & $\mathrm{Fi}$ \\
\hline 20. & Pihri chara & Mecardonia procumbens (Mill.) Small & Scrophulariaceae & $\mathrm{H}$ & Fo \\
\hline 21. & Ratolia & Phyla nodiflora (L.) Greene & Verbenaceae & $\mathrm{H}$ & $\mathrm{V}$ \\
\hline 22. & Kharatti & Sida acuta Burm.f. & Malvaceae & $\mathrm{H}$ & M \\
\hline 23. & Kangni & Setaria pumila (Poir.) Roem. \& Schult. & Poaceae & G & Fo \\
\hline 24. & Bharbhusi & Eragrostis tenella (L.) P.Beauv. ex Roem. \& Schult. & Poaceae & G & Fo \\
\hline 25. & Soli & Aeschynomene americana $\mathrm{L}$. & Leguminosae & $\mathrm{H}$ & $\mathrm{Gm}$ \\
\hline 26 & Patthar choor & Plectranthus mollis (Aiton) Spreng. & Lamiaceae & $\mathrm{H}$ & M \\
\hline 27. & Bariyari & Sida cordata (Burm.f.) Borss.Waalk. & Malvaceae & $\mathrm{H}$ & M \\
\hline 28. & Hirankhuri & Emilia sonchifolia (L.) DC. ex DC. & Asteraceae & $\mathrm{H}$ & M \\
\hline 29. & Badranj boya & Nepeta cataria $\mathrm{L}$. & Lamiaceae & $\mathrm{H}$ & M \\
\hline 30. & Kevkand & Dioscorea bulbifera L. & Dioscoreaceae & $\mathrm{C}$ & M \\
\hline 31. & Kali mushli & Curculigo orchioides Gaertn & Agavaceae & $\mathrm{T}$ & M \\
\hline
\end{tabular}




\begin{tabular}{|c|c|c|c|c|c|}
\hline S. No & common name & Scientific name & Family & Habit & uses \\
\hline 32. & Tinpaniya & Oxalis corniculata $\mathrm{L}$. & Oxalidaceae & $\mathrm{H}$ & $M \& V$ \\
\hline 33. & Maskani & Evolvulus nuтmularius (L.) & Convolvulaceae & $\mathrm{H}$ & $\mathrm{M}$ \\
\hline 34. & Chanchu & Corchorus fascicularis Lam. & Tiliaceae & $\mathrm{H}$ & $\mathrm{Fi}$ \\
\hline 35. & Kena & Commelina diffusa Burm.f. & Commelinaceae & $\mathrm{H}$ & M \\
\hline 36. & Kharmor & Rungia pectinata (L.) Nees & Acanthaceae & $\mathrm{H}$ & M \\
\hline 37. & Ghueen & Fimbristylis littoralis Gaudich. & Cyperceae & $\mathrm{H}$ & M \\
\hline 38. & Nagar motha & Cyperus gracilis $\mathrm{R} . \mathrm{Br}$. & Poaceae & G & Fo \\
\hline 39. & Bufalo grass & Paspalum conjugatum P.J.Bergius & Poaceae & G & Fo \\
\hline 40. & Baiga sikiyab & Digitaria divaricatissima (R.Br.) Hughes & Poaceae & G & Fo \\
\hline 41. & Jangli marua & Eleusine indica (L.) Gaertn. & Poaceae & G & Fo \\
\hline 42. & Dokar bel & Vitis carnosa (Lam.) Wall. & Vitaceae & $\mathrm{H}$ & M \\
\hline 43. & Chui mui & Mimosa pudica $\mathrm{L}$. & Fabaceae & $\mathrm{H}$ & M \\
\hline 44. & Nuniya bhaji & Portulaca oleracea $\mathrm{L}$. & Portulaceae & $\mathrm{H}$ & $\mathrm{V}$ \\
\hline 45. & Kanthkari & Solanum xanthocarpum Schrad. \& H. Wendl. & Solanaceae & $\mathrm{H}$ & M \\
\hline 46. & Jungli sama & Echinochloa colona (L.) Link & Poaceae & G & $\mathrm{Ge}$ \\
\hline 47. & Amti & Solanum nodiflorum Jacq. & Solanaceae & $\mathrm{H}$ & $\mathrm{V}$ \\
\hline 48. & Chirchita & Achyranthes aspera $\mathrm{L}$. & Amaranthaceae & $\mathrm{H}$ & M \\
\hline 49. & Ghooma & Leucas aspera (Willd.) Link & Lamiaceae & $\mathrm{H}$ & V \\
\hline 50. & Kaniya kanda & Dioscorea oppositifolia L. & Dioscoreaceae & $\mathrm{C}$ & $\mathrm{M}$ \\
\hline 51. & Chench & Corchorus trilocularis L. & Tiliaceae & $\mathrm{H}$ & $\mathrm{V}$ \\
\hline 52. & Chanahur & Marsdenia tenacissima (Roxb.) Moon & Asclepiadaceae & $\mathrm{H}$ & $\mathrm{V}$ \\
\hline 53. & Van rai & Blumeopsis flava (DC.) Gagnep. & Asteraceae & $\mathrm{H}$ & M \\
\hline 54. & Tikhur & Curcuma angustifolia Roxb. & Zingiberaceae & $\mathrm{T}$ & M \\
\hline 55. & Bhui amla & Phyllanthus niruri L. & Euphorbiaceae & $\mathrm{H}$ & M \\
\hline 56. & Salparni & Desmodium gangeticum (L.) DC. & Fabaceae & $\mathrm{H}$ & M \\
\hline 57. & Satawar & Asparagus racemosus Willd. & Liliaceae & $\mathrm{H}$ & M \\
\hline 58. & Haddi mushli & Chlorophytum borivilianum Santapau \& R.R.Fern. & Asparangaceae & $\mathrm{T}$ & M \\
\hline 59. & Datura & Datura metel L. & Solanaceae & $\mathrm{H}$ & $\mathrm{M}, \mathrm{Ru}$ \\
\hline 60. & Gajar ghas & Parthenium hysterophorus $\mathrm{L}$. & Asteraceae & $\mathrm{H}$ & M \\
\hline 61. & Badi dudhi & Euphorbia hirta L. & Euphorbiaceae & $\mathrm{H}$ & M \\
\hline 62. & Chhoti dudhi & Euphorbia macrophylla Pax & Euphorbiaceae & $\mathrm{H}$ & M \\
\hline 63. & Bara & Flemingia chappar Benth. & Fabaceae & $\mathrm{H}$ & Lac \\
\hline 64. & Bedarikand & Coccinia grandis (L.) Voigt & Cucurbitaceae & $\mathrm{C}$ & $\mathrm{E}$ \\
\hline 65. & Jungli san & Crotalaria spectabilis Roth & Fabaceae & $\mathrm{H}$ & M \\
\hline 66. & Kalihari & Gloriosa superba L. & Colchicaceae & $\mathrm{C}$ & $\mathrm{M}$ \\
\hline 67. & Kheksa & Momordica dioica Roxb & Cucurbitaceae & $\mathrm{C}$ & $\mathrm{V}$ \\
\hline 68. & Karmata & Ipomoea aquatica Forssk. & Convalvulaceae & $\mathrm{H}$ & $\mathrm{V}$ \\
\hline 69. & Jungle kevanch & Mucuna pruriens $(L) D C$. & Papilionaceae & $\mathrm{H}$ & M \\
\hline 70. & Jangli pyaj & Urginea indica (Roxb.) Kunth & Liliaceae & $\mathrm{H}$ & M \\
\hline 71. & Chirula & Aerva lanata (L.) Juss. & Amaranthaceae & $\mathrm{H}$ & M \\
\hline 72. & Chirinya & $\begin{array}{l}\text { Peristrophe roxburghiana (Roem. \& Schult.) } \\
\text { Bremek. }\end{array}$ & Acanthacea & $\mathrm{H}$ & M \\
\hline 73. & Garundi & Alternanthera sessilis (L.) R.Br. ex DC. & Amaranthaceae & $\mathrm{H}$ & M \\
\hline 74. & Jungli rye & Sisymbrium nigrum (L.) Prantl & Cruciferae & $\mathrm{H}$ & $\mathrm{V}$ \\
\hline 75. & Jangli Tulsi & Ocimum gratissimum $\mathrm{L}$. & Lamiaceae & $\mathrm{H}$ & M \\
\hline 76. & Chirpoti & Physalis minima $\mathrm{L}$. & Solanaceae & $\mathrm{H}$ & $\mathrm{Fe}$ \\
\hline 77. & Sarpgandha & Rauvolfia serpentina (L.) Benth. ex Kurz & Apocynaceae & $\mathrm{H}$ & M \\
\hline 78. & Sadabahar & Catharanthus roseus (L.) G.Don & Apocynaceae & $\mathrm{H}$ & M \\
\hline 79. & Brahmi & Bacopa monnieri (L.) Wettst. & Plantaginaceae & $\mathrm{H}$ & M \\
\hline 80. & Tulsi & Ocimum sanctum $\mathrm{L}$. & Lamiaceae & $\mathrm{H}$ & $\mathrm{M}, \mathrm{Ru}$ \\
\hline 81. & Chirayta & Swertia alba T.N. Ho \& S.W. Liu & Gentianaceae & $\mathrm{H}$ & $\mathrm{M}$ \\
\hline 82. & Aswagandha & Withania somnifera (L.) Dunal & Solanaceae & $\mathrm{T}$ & $\mathrm{M}$ \\
\hline 83. & Chand kal & Macaranga peltata (Roxb.) Müll.Arg. & Euphorbiaceae & $\mathrm{H}$ & $\mathrm{M}$ \\
\hline 84. & Chaulai & Amaranthus spinosus L. & Amaranthaceae & $\mathrm{H}$ & $\mathrm{V}$ \\
\hline 85. & Tiger lily & Belamcanda chinensis (L.) DC. & Iridaceae & $\mathrm{H}$ & M \\
\hline 86. & Bisakhpara & Boerhavia procumbens Banks ex Roxb. & Nyctaginaceae & $\mathrm{H}$ & $\mathrm{V}$ \\
\hline 87. & Mandukparni & Centella asiatica (L.) Urb. & Apiaceae & $\mathrm{H}$ & M \\
\hline 88. & Ghuia & Colocasia esculenta (L.) Schott. & Araceae & $\mathrm{H}$ & V \\
\hline 89. & Kev kand & Costus speciosus (J.Koenig) Sm. & Zingiberaceae & $\mathrm{T}$ & M \\
\hline 90. & Amahaldi & Curcuma amada Roxb. & Zingiberaceae & $\mathrm{T}$ & M \\
\hline 91. & jungli dhania & Eryngium foetidum $\mathrm{L}$. & Apiaceae & $\mathrm{H}$ & $\mathrm{V}$ \\
\hline 92. & Sitab & Ruta graveolens $\mathrm{L}$. & Rutaceae & $\mathrm{H}$ & M \\
\hline 93. & Mameera & Thalictrum foliolosum DC. & Rananculaceae & $\mathrm{H}$ & M \\
\hline 94. & Bathua bhaaji & Chenopodium album $\mathrm{L}$. & Chenopodiaceae & $\mathrm{H}$ & $\mathrm{V}$ \\
\hline
\end{tabular}

Legends: F: Food, H: Herb, T: Tuber, C: Climber, G: Grass, M: Medicinal use, V: Vegetable, Fo: Fodder, Fe: Fruit edible, Ru: Religious use, E: Edible, Ge: Grain edible and Gm: Grain edible 
Table 3. Traditionally used tree species by local people in aabr.

\begin{tabular}{|c|c|c|c|c|c|}
\hline 8 & Common name & Scientific name & Family & Parts used & Uses \\
\hline 1. & Bel & Aegle marmelos & Rutaceae & Fruit Leaf & Edible, Medicinal, Religious purpose \\
\hline 2. & Dhabda & Anogeissus latifolia & Combretaceae & Stem Resin & $\begin{array}{l}\text { House construction Fuel wood } \\
\text { Agriculture implement Selling }\end{array}$ \\
\hline 3. & Mohline & Bauhinia purpurea & Caesalpiniaceae & Leaf Flower & Cup and plate making Medicinal \\
\hline 4. & Semel & Bombax ceiba & Malvaceae & Fruit Flower & Medicine Edible \\
\hline 5. & Salei & Boswellia serrata Roxb. & Burseraceae & Resin & Medicine \\
\hline 6. & Chironji & Buchanania lanzan & Anacardiaceae & Fruit seed & Edible Edible \\
\hline 7. & Khakra & Butea monosperma & Fabaceae & Leaf & Cup and plate making \\
\hline 8. & Kumbhi & Careya arborea & Lecythidaceae & Bark & Fish poisoning \\
\hline 9. & Amaltash & Casia fistula & caesalpinaceae & Fruit & Medicinal. \\
\hline 10. & Mahalimb & Cedrela toona Roxb. & Meliaceae & Stem & Furniture \\
\hline 11. & Ghiriha & Chloroxylon swietenia & Rutaceae & Stem Bark & $\begin{array}{l}\text { House Construction Agricultural implements Fuel wood } \\
\text { Fish poison }\end{array}$ \\
\hline 12. & Karra & Cleistanthus collinus & Euphorbiaceae & Stem & Furniture \\
\hline 13. & Sita phal & Custard apple & Annonaceae & Fruit Stem & $\begin{array}{l}\text { Edible House Construction Agricultural implements } \\
\text { Fuel wood }\end{array}$ \\
\hline 14. & Shisham & Dalbergia sisoo & Leguminosae & Stem Leaf & $\begin{array}{l}\text { House Construction Agricultural implements Fuel wood } \\
\text { Medicinal }\end{array}$ \\
\hline 15. & Gulmohar & Delonix regia & Leguminosae & Stem & Fuel wood \\
\hline 16. & Dhoben & Dillenia pentagyna Roxb. & Dilleniaceae & Root & Medicinal \\
\hline 17. & Tendu & Diospyros melanoxylon Roxb. & Ebenaceae & Fruit Leaf & Edible (When ripe) Selling \\
\hline 18. & Bargad & Ficus benghalensis & Moraceae & Fruit & Edible \\
\hline 19. & Peepal & Ficus religiosa & Moraceae & $\begin{array}{l}\text { Whole tree } \\
\text { Fruit Leaf }\end{array}$ & Religious Edible Fodder \\
\hline 20. & Kekad & Garugapinnata Roxb. & Burseraceae & Stem & Agricultural implements \\
\hline 21. & Lendia & Lagerstroemia parviflora Roxb. & Lythraceae & Stem & Firewood Boundary wall making \\
\hline 22. & Maida & Litsea sebifera & Lauraceae & Bark & Medicinal \\
\hline 23. & Mahua/ Guli & Madhuca indica & Sapotaceae & $\begin{array}{l}\text { Flower Fruit } \\
\text { Leaf }\end{array}$ & Edible after cooking Liquor preparation Oil Religious \\
\hline 24. & Aam & Mangifera indica & Anacardiaaceae & Fruit Seed & Edible Edible, medicinal \\
\hline 25. & Kem & Mitragyna parviflora & Rubiaceae & Leafy branch & Cultural uses \\
\hline 26. & Munga & Moringa pterygosperma Gaertn. & Moringaceae & Leaf Fruit & Edible Edible \\
\hline 27. & Amla & Phyllanthus emblica & Euphorbiaceae & Fruit Leaf & Edible and medicinal Cultural and medicinal \\
\hline 28. & Kanji & Pongamia pinnata & Fabaceae & Fruit & Oil extraction \\
\hline 29. & Bija & Pterocarpus marsupium Roxb. & Faabaceae & Stem & House construction Furniture \\
\hline 30. & Kusum & Schleichera trijuga Willd. & Sapindaceae & Fruit & Edible \\
\hline 31. & Bhelwa & Semecarpusanacardium & Anacardiaceae & Fruit & Edible, Medicinal \\
\hline 32. & Sarei & Shorea robusta Gaertn. & Dipterocarpaceae & Stem & $\begin{array}{l}\text { House construction Furniture Fuel wood } \\
\text { Plough Making Cultural, Tooth brush, oil }\end{array}$ \\
\hline 33. & Gulhar/kullu & Sterculia urens & Sterculiaceae & Resin Bark & Medicinal Rope making \\
\hline 34. & Jamun & Syzygium cumini & Myrtaceae & $\begin{array}{l}\text { Stem Fruit } \\
\text { Leaf }\end{array}$ & Cultural Edible Cultural and medicinal \\
\hline 35. & Emli & Tamarindus indica & Caesalpiniaceae & Fruit & Edible, Pickle preparation, Medicinal, Selling \\
\hline 36. & Sagaun & Tectona grandis & Lamiaceae & Stem Leaf & House construction, Furniture Cultural. Dona making \\
\hline 37. & Arjun & Terminalia arjuna & Combretaceae & Stem & Firewood, House construction \\
\hline 38. & Beheda & Terminalia bellirica & Combretaceae & Fruit & Medicinal (Digestive) \\
\hline 39. & Harra & Terminalia chebula & Combretaceae & Fruit & Medicinal (Digestive) \\
\hline 40. & Saja & Terminalia tomentosa & Combretaceae & Stem & $\begin{array}{l}\text { House construction Fuel wood } \\
\text { Used during marriage }\end{array}$ \\
\hline
\end{tabular}

Table 4. Traditionally used Shrub species by local people in AABR.

\begin{tabular}{|c|c|c|c|c|c|}
\hline S. No. & Common name & Scientific name & Family & Parts used & Uses \\
\hline 1. & Ghughch & Abrus precatorius & Fabaceae & Leaves & Mouth freshener \\
\hline 2. & Bans & Bambusa bamboo & Poaceae & Seeds & mix into flour \\
\hline 3. & Chakor & Cassia tora & Caesalpiniaceae & Pod and seed & Vegetable \\
\hline 4. & Ratan jot & Jathropa curcus & Euphorbiaceae & Seed Whole plant & Substitute of candle Bio-fencing \\
\hline 5. & Lantana & Lantana camara & Verbenaceae & Ripen fruits Whole plant & Edible Bio-fencing \\
\hline 6. & Khajuri & Phoenix sylvestris & Arecaceae & Ripen fruits & Edible. \\
\hline 7. & Mainhar & Randia dumetorum & Rubiaceae & Leaf Root & Vegetable Medicinal \\
\hline 9. & Nirgundi & Vitex nigundo & Verbenaceae & Leaf & Medicinal \\
\hline 10. & Ber & Zizipus zilopyrus & Rhamnaceae & Fruit & Edible \\
\hline 11. & Aak & Calotropis gagentia & Musaceae & Leaf \& flower & Offer to god \\
\hline
\end{tabular}




\begin{tabular}{llllll}
\hline S. No. & Common name & Scientific name & Family & Parts used & Uses \\
\hline 12. & Banana & Musa paradisca & Family & Whole tree & Religious use \\
13. & Mehandi & Lawsonia irnemis & Lythraceae & Leaf & Dye \\
\hline
\end{tabular}

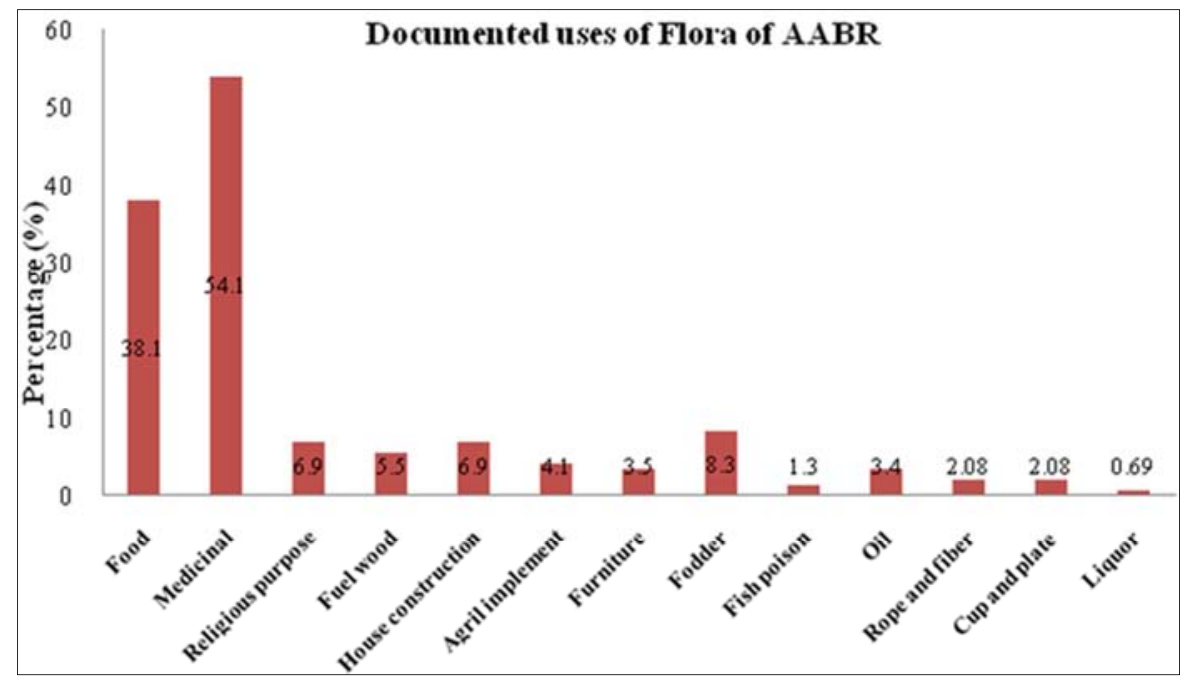

Figure 2. Traditionally used flora of AABR for diverse usufructs by local people.

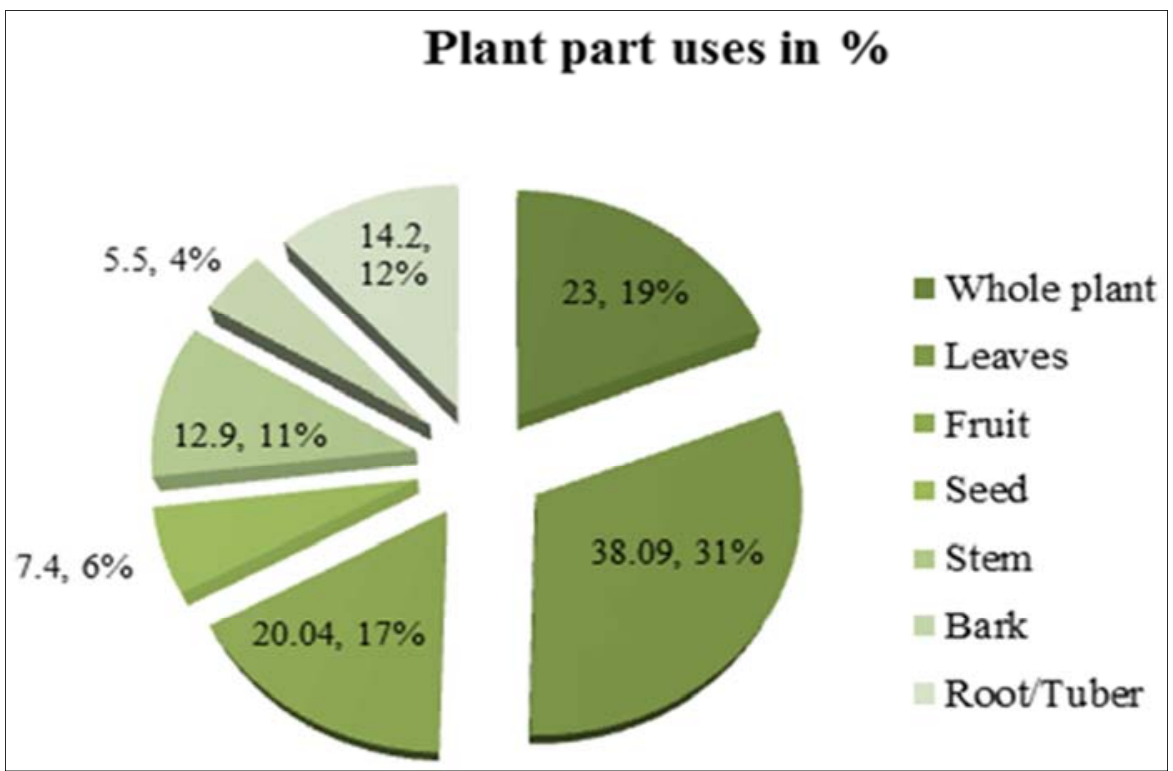

Figure 3. The utlis ation pattern of plant part used by local people of AABR.

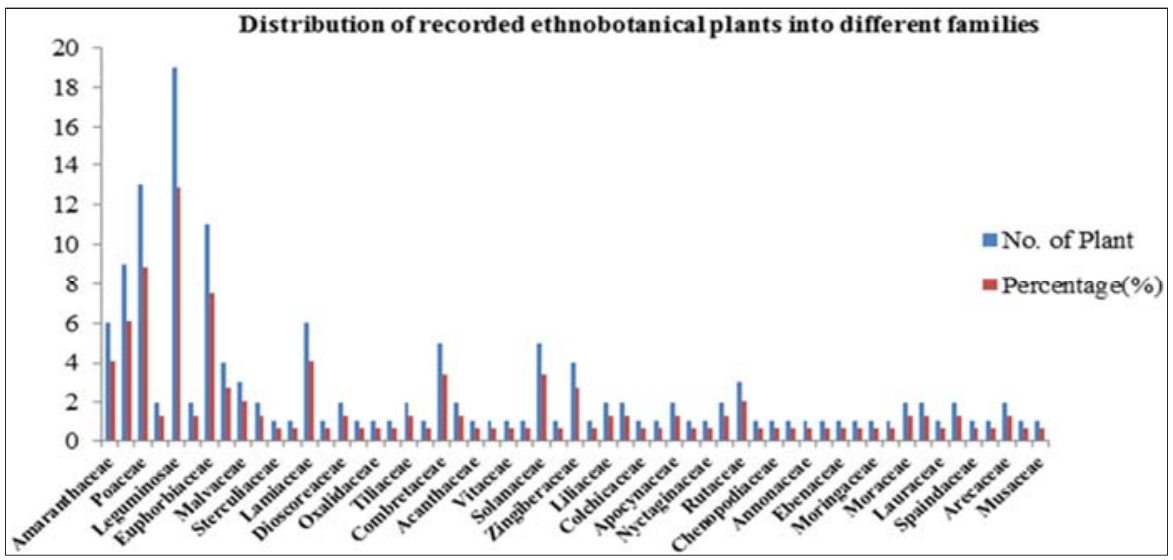

Figure 4. Distribution of recorded ethnobotanical plants intobotanical families. 
Of the reported growth form of flora used by local people of $\mathrm{AABR}$, tree \& herbs make up the highest ratio of locally consumed species comprising $27.2 \%$ and $48.9 \%$ respectively (Figure 5). The present study on the traditional use of flora support by many earlier workers in India and elsewhere in world (Dlamini et al. 2011; Uprety et al. 2012; Panda, 2014; Dutta, 2015). Some of the studied floras are also used in Pachmarhi Biosphere Reserve and other parts of country (Sinha et al. 2005; Rout, 2007; Kala, 2009). Similar findings were laid by Bharucha et al.2010, Dobriyal, et al, 2015. During the study we have observed that the products (forest) consumed or sold by these communities also have same use worldwide however, some of them are unique here, according to their (tribal's) indigenous knowledge and traditional uses like religious use, use of plant according to food habit and medicinal use. During the survey it was also observed that the Baiga is most primitive tribal's group of AABR and have enormous knowledge about the use of forest products. Due to modernization, like agriculture settlement (Pei et al. 2009), less interest of young generation (Panda, 2014) a drastic change in food styles (Negi et al. 2015) and very few documentation, the traditional knowledge remain in collective memory of the old faces and disappearing after the death of them.

Table 5. Listing of culturally associated flora (CAF) of AABR.

\begin{tabular}{llll}
\hline S.N & $\begin{array}{l}\text { Local } \\
\text { name }\end{array}$ & Scientific name & Culturally uses \\
\hline 1 & Datura & Datura metel & Flower offer to god \\
2 & Tulsi & Ocimum sanctum & Treated Prosperity of home \\
3 & Bel & Aegle marmelos & Leaf offer to god \\
\hline
\end{tabular}

\begin{tabular}{llll}
\hline S.N & $\begin{array}{l}\text { Local } \\
\text { name }\end{array}$ & Scientific name & Culturally uses \\
\hline 4 & Mahua & Madhuca indica L & $\begin{array}{l}\text { Look like as source of } \\
\text { subsistence of life }\end{array}$ \\
5 & Peepal & Ficus religiosa & Whole tree \\
6 & Jamun & Syzygium cumini $($ L. $)$ & Leaf used in festival \\
7 & Aak & Calotropis gagentia & Leaf \& flower offer to god \\
8 & Aonla & Phyllanthus emblica & Whole tree \\
9 & Banana & Musaparadisiacal & Whole tree \\
10 & Mehandi & Lawsonia irnemis & Leaf dye used in marriage \\
\hline
\end{tabular}

The traditional knowledge, religious uses and cultural association of these communities with the natural resources serve as a conservation attitude. The same results were found in many earlier studies carried out in India (Pandey, 1997; Kala, 2009; Negi et al. 2015) and other parts of world (Gunatilleke et al. 1993; Anderson et al. 2002; Rist et al.2008; Pie et al. 2009). These communities symbiotically linked with these natural resources and have a strong perception that they could not exist without forest in nature. They fulfill their need from forest and other hand they conserve the forest. During the study we have observed luxuriant forest growth in interior areas of biosphere. Several other studies all over the world found same result that the area which have lesser impact of outsider, have well maintained forest (Liu et al. 2000; Xu, 2003; Pei et al. 2007 \& 2009). The persistence of the luxuriant growth of the forest in the interior areas of biosphere are the result of several unknown customs and tradition of these societies. Before collection and consumption of these floras, mainly culturally associated flora (CAF) they perform certain rituals and practices which serve as a conservation ethics for natural resources (Table 5).

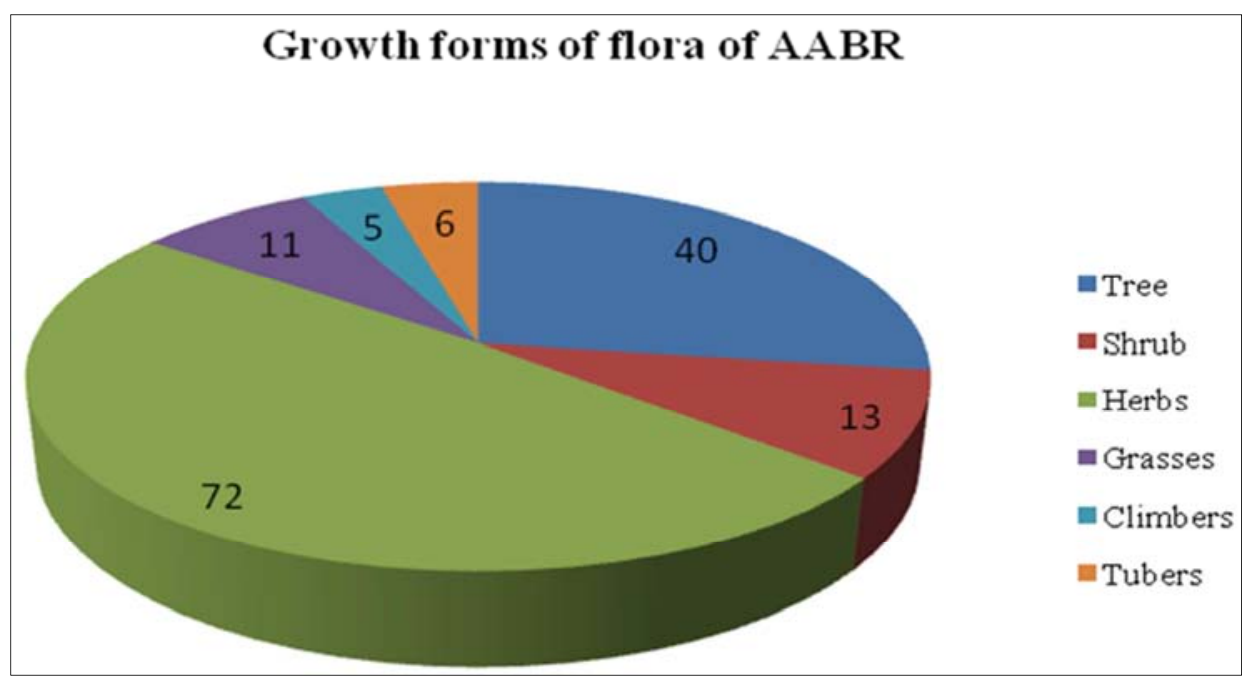

Figure 5. Reported growth forms of flora used by local people of $A A B R$.

\subsection{Aboriginals Assist to Biodiversity Conservation and Climate Protection}

Tribal communities play leading role in conservation of biodiversity through their vast knowledge about the use of concerned flora and also association of flora with their culture. The conservation specially in-situ addressed to climate protection everywhere in the world. Recently
IPCC quoted that these are the communities which are in frontline of climate variability but due to their conservation tactics they easily takeoff from these variability. There are plenty of species which are conserved by them through their ritual association, due to their food habit and traditional value or commercial value of plants and principles of ethnoforestry among these communities (Table 6). 
Table 6. List of species conserved on the name of god/goddess, on scared grooves and for edible purpose by tribal communities.

\begin{tabular}{lllll}
\hline S.No. & Local Name & Scientific name & Family Name & Remark \\
\hline 1 & Aam & Mangifera indica & Anacardiaceae & Lord Vidhyadhara \\
2 & Arjun & Terminalia arjuna & Combertaceae & Lord Brhma \\
3 & Nibu & Citrus medica & Rutaceae & Lord Brahaspati \\
4 & Bilva & Aegle marmelos & Rutaceae & Lord Shiva \\
5 & Nimba & Azadiracta indica & Meliaceae & Goddess Sheetla mata \\
6 & Basil & Ocimum sanctum & Lamiaceae & Goddess Lakshmi \\
7 & Baka & Sesbania grandiflora & Fabaceae & Lord Narayan \\
8 & Karavira & Nerium indicum & Apocynaceae & Lord Ganesh \\
9 & Nilapadma & Nelumbi nucifera & Nymphaceae & Goddess Ambika \\
10 & Madar & Calotropis gigantean & Asclepiadeceae & Lord Shiva \\
11 & Chirchita & Achyranthus aspera & Amaranthaceae & Tender shoots as vegetable \\
12 & Molian leaf & Bauhinia purpurea & Ceasalpiniaceae & Leaves, flower, seeds as vegetable \\
13 & Dudhia aru & Dioscorea alta & Dioscoreaceae & Tubers used as vegetable \\
14 & Palas & Butea monosperma & Fabaceae & Conserved on scared grooves \\
15 & Slai & Boswellia serrata & Burseraceae & Conserved on scared grooves \\
16 & Madar & Calotropis gigantean & Asclepiadeceae & Conserved on scared grooves \\
17 & Bamboo & Bambusa arudinacea & Poaceae & Conserved on scared grooves \\
18 & Sarpgandha & Ravuolfia serpentina & Apocynaceae & Conserved on scared grooves \\
19 & Aam & Mangifera indica & Anacardiaceae & Conserved on scared grooves \\
20 & Peepal & Ficus religiosa & Moraceae & Conserved on scared grooves \\
\hline
\end{tabular}

Source: Jain, S.K. 1996. Ethnobiology in Human Welfare, Deep Publication, New Delhi.

Table 7. Forest products sale locally or in regional market by local people of AABR.

\begin{tabular}{|c|c|c|c|c|c|c|c|}
\hline S No & Forest products & $\begin{array}{l}\text { Season of } \\
\text { collection }\end{array}$ & Duration (month) & Importance & Market value (in Rs.) & $\begin{array}{l}\text { Multiple use of } \\
\text { the product }\end{array}$ & Remark \\
\hline 1 & Sal leaf & $\begin{array}{l}\text { All season except } \\
\text { leaf fall duration }\end{array}$ & 8 & & $\begin{array}{l}\text { Rs. } 15 / 1000 \\
\text { plates }\end{array}$ & & 5 \\
\hline 2 & Sal seed & May- June & 2 & & Rs. $12 / \mathrm{Kg}$ & $\mathrm{X}$ & 4 \\
\hline 3 & Fuel wood & All season & 12 & & Rs. 90/ bundle (4 Kg) & & 3 \\
\hline 4 & mushroom & July- September & 3 & & Rs. $150-200 / \mathrm{kg}$ & & 1 \\
\hline 5 & Mahua flower & May-June & 2 & & Rs. $30-40 / \mathrm{kg}$ & & 2 \\
\hline 6 & $\begin{array}{l}\text { Bamboo kareel } \\
\text { Van karela }\end{array}$ & Rainy season & 3 & & Rs. $70-80 / \mathrm{kg}$ & & 2 \\
\hline 7 & $\begin{array}{l}\text { (Momordica } \\
\text { charantia) }\end{array}$ & Rainy season & 3 & & Rs. $15-20 / \mathrm{kg}$ & & 3 \\
\hline 8 & mainahar & Rainy season & 2 & & Rs. $10-15 / \mathrm{kg}$ & & 3 \\
\hline 9 & Uhar kuhar bhaji & All season & 12 & & Rs. $15-20 / \mathrm{kg}$ & & 3 \\
\hline 10 & $\begin{array}{l}\text { Munga(morina } \\
\text { olieferaa) }\end{array}$ & $\begin{array}{l}\text { Late summer to } \\
\text { rainy season }\end{array}$ & $3-4$ & & Rs.10-15 & & 3 \\
\hline 11 & Murraya koenigii & All season & $8-12$ & & Rs. $5-7 / \mathrm{kg}$ & & 5 \\
\hline 12 & Sal dhupa & Rainy season & $1-2$ & & Rs. $90-100 / \mathrm{kg}$ & & 1 \\
\hline 13 & Bahunia leaf & Rainy season & $3-4$ & & Rs. $12 / \mathrm{kg}$ & & 3 \\
\hline 14 & Aonla fruit & Late rainy season & $2-3$ & & Rs. $30-40 / \mathrm{kg}$ & & 2 \\
\hline 15 & Cassia tora & $\begin{array}{l}\text { Summer to late } \\
\text { rainy season }\end{array}$ & $6-8$ & & R.s15-20/kg & & 2 \\
\hline
\end{tabular}

Data Source: Based on Questionnaire Survey of forest villagers of AABR.

*Rank: 1-Highly important, 2- Important 3-Modretly important, 4- Less important, 5- Very less important, according to market price

The forest products collected form forest or nearby village are sale in local or regional market by the local people of AABR (Table 7). The forest products play important role in the viability and survival of tribal and non tribal communities of AABR. Forest dwellers of AABR collected these products from forest or nearby village area for household consumption and also for sale in the local market. Many wild edible plant species are found to be sold in the local markets particularly by poor and economically marginalized families, thereby generating a supplementary income to their household economy (Panda, 2014). Many earlier workers, worldwide also reported that forest is a source of subsistence of these isolated communities (Chittaranjan, 2005; Colchester et al. 2006; Muhaammed et al. 2010; Khera, 2016). The important role of forest products is not only in meeting the subsistence needs but also in poverty alleviation (FAO, 1995). The forest products are very economically important in point of view of subsistence of local people of BR. In case of these indigenous communities, the major factor of economics such as production, consumption and distribution are closely associated with forest (Chittaranjan, 2005).These hidden societies of the word have great assumption that the natural resources have only means of survival of our lives. Utilization of forest resources is a prerequisite for the 
livelihood of remote villagers who do not have many other alternative source of income (Yeo-Chang, 2009). Forest and forest resources, predominantly minor forest products (vegetables, fruit, medicinal, gum, fuel wood, seeds, grasses, and even soil) occupy an important role in continued existence of tribal's life (Aboriginal communities) in AABR or elsewhere in world.

\section{Conclusion}

The results of this study has revealed that indigenous traditional knowledge on the use of flora like edible food, vegetables and cure to certain diseases is still practiced by the local communities of AABR. Beside, these uses the forest is also source of income, they sale some products in local or regional market. The local communities very well known to the uses of ethnobotanicals which are little known and unexplored to modern societies. So there is a strong need of documentation and conservation of these floras for as a source of food, medicine, and sustained income at a time of scarcity.

\section{Acknowledgement}

The authors express deep sense of gratitude to the members of tribal communities for providing useful information in favor of indigenous knowledge of local flora and also extended help in identification of rare flora of the study area.

\section{References}

[1] Anderson, P. J. and Putz, F. E., 2002. Harvesting and conservation: are both possible for the palm, Iriartea deltoidea? Forest Ecol. Manage. 170, 271-283.

[2] Balemie, K. and Kebebew, F. 2006. Ethnobotanical study of wild edible plants in Derashe and Kucha Districts, South Ethiopia. J Ethnobiol Ethnomed, 2:53-61. http://dx.doi.org/10.1186/1746-4269-2-53.

[3] Bharucha, Z. and Pretty, J. 2010. The roles and values of wild foods in agricultural systems. Phil Trans Royal Soc B., 365: 2913-2926. http://dx.doi.org/10.1098/rstb.2010.0123.

[4] Bonet, M. A. and Vallès, J. 2002. Use of non-crop food vascular plants in Montseny biosphere reserve (Catalonia, Iberian Peninsula). Inter J, Food Sci Nutr, 53: 225-248.

[5] Burgi, M., Gimmi, U. and Stuber, M. 2013. Assessing traditional knowledge on forest uses to understand forest ecosystem dynamics. Forest Ecology and Management. 289, 115- 122.

[6] Chittaranjan, K. P. 2005. Food and agriculture organization (FAO) report. A text book "Forest government and tribe."

[7] Colchester, M. 2006. Justice in the forest: Rural lively hoods and forest law enfo: fores perspectives, CIFOR, Indonesia.

[8] Cunningham, A. B. 2001. Applied ethnobotany, people wild plant use and conservation. Sterling, VA, London, Earth Scan
Publication Ltd.

[9] Dlamini, C. S. and Geldenhuys, C. J. 2011. A resource survey for medicinal and edible plant species in the four ecological zones of rural Swaziland. Journal of Geography and Regional Planning, 4(9): 557-555.

[10] Dobriyal, Manmohan J. R. and Dobriyal, Ranjana (2015) Non wood forest produce: an option for ethnic food and nutritional security in India. International Journal of Forest Usufructs Management, 15 (1): 17-37.

[11] Dutta, B. 2015.Food and medicinal values of certain species of Dioscorea with special reference to Assam. Journal of Pharmacognosy and Phytochemistry, 3(4): 15-18.

[12] Ertug, F. 2004. Wild edible plants of the Bodrum Area (Mugla, Turkey). Turk J Bot, 28: 161-174.

[13] Food and Agriculture Organization of the United Nations (FAO) 2004. Annual Report: The state of food insecurity in the world, monitoring the progress towards the world food summit and millennium development goals, Rome.

[14] Gadgil, M., Berkes, F. and Folke, C. (1993). Indigenous knowledge for biodiversity conservation. Ambio, 22: 151-156.

[15] Grivetti, L. E. and Ogle, B. M. 2000. Value of traditional foods in meeting macro and micronutrient needs: the wild plant connection, Nutrition Research Reviews, 13,31-46.

[16] Guarrera, P. M. 2003. Food medicine and minor nourishment in the folk traditions of Central Italy (Marche, Abruzzo and Latium). Fitoterapia, 74: 515-544. http://dx. doi. org/10.1016/S0367-326X (03)00122-9.

[17] Gunatilleke, I. A. U. N., Gunatilleke, C. V. S. and Abeygunawardena, P., 1993. Interdisciplinary research towards management of non-timber forest resources in lowland rain forests of Sri Lanka. Econ. Bot. 47 (3), 282-290.

[18] Jain, S. K. 1996. Ethnobiology in Human Welfare, Deep Publication, New Delhi. Pp:519-520.

[19] Javier, T., Manuel, P. S. A. and Ramón, M. 2006. Ethnobotanical review of wild edible plants in Spain. Bot J Linn Soc, 152:27-71. http://dx.doi.org/10.1111/j.10958339.2006.00549.x.

[20] Khera, A. 2016. Poverty alleviation through non wood forest products in Madhya Pradesh. Journal of tropical forestry, 32 (II).

[21] Liu, A., Pei, S. and Chen, S., 2000a. Yi nationality's sacred groves and biodiversity conservation in Chuxiong, Yunnan. Chin. J. Appl. Ecol. 11 (4), 489-492.

[22] Muhaammed, N. V. D. and Sheeladitya, R. C. 2010. Livelihood pattern and forest dependence of the major tribes in Rangamati Bangladesh. Shinshu University International symposium 20-02-2010.

[23] Negi, P. S. and Subramani, S. P. 2015. Wild edible plant genetic resources for sustainable food security and livelihood of Kinnaur district, Himachal Pradesh, India. Int J Conserv Sci, 6 (4): 657-668.

[24] Ogle, B. M., Dung, N. N. X., Do, T.T.and Hambraeus, L. 2001. The contribution of wild vegetables to micronutrient intakes among women. An example from the Mekong Delta, Vietnam. Ecol Food Nutr, 40: 159184.http://dx.doi.org/10.1080/03670244.2001.9991646. 
[25] Ogoye-Ndegwa, C. and Aagaard-Hansen, J. 2003. Traditional gathering of wild vegetables among the Luo of Western Kenya - a nutritional anthropology project. Ecol Food Nutr, 42: 6989. http://dx.doi.org/10.1080/03670240303114.

[26] Panda, T. 2014. Traditional knowledge on wild edible plants as livelihood food in Odisha, India. Journal of Biology and Earth Sciences, 4 (2): B144-B159

[27] Pandey, A. K. 1997. Tribal Society in India, Mannak Publication, New Delhi.

[28] Pei, S. and Huai, H. 2007. Ethnobotany, Shanghai Science and Technology Press, Shanghai, China.

[29] Pei, S., Zhang, G. and Huai, H. 2009. Application of traditional knowledge in forest management: Ethnobotanical indicators of sustainable forest use. Forest Ecology and Management. 257, 2017-2021.

[30] Rist, L., Shaanker, R. U., Milner-Gulland, E. J. and Ghazoul, J., 2008. Managing mistletoes: the value of local practices for a non-timber forest resource. Forest Ecol. Manage. 255, 16841691.

[31] Riter, E. and Dauksta, D. (2013). Human- forest relationship: Ancient values in modern perspectives. Environment development and sustainability, 15, 645-662.
[32] Rout, S. D. 2007. Ethnobotany of diversified wild edible fruit plants in Similipal Biosphere Reserve. Ethnobotany,19: 137139.

[33] Sinha, R. and Larka, V. 2005. Wild tribal food plants of Orissa. Ind J Trad Know, 4(3): 246-252.

[34] Tanji, A. and Nassif F. 1995. Edible weeds in Morocco. Weed Technol, 9: 617-620.

[35] Tardío, J., Pascual, H. and Morales, R. 2005. Wild food plants traditionally used in the province of Madrid. Econ Bot,59: 122-136.

[36] Uprety, Y., Poudel, R. C., Shrestha, K. K., Rajbhandary, S., Tiwari, N. N., Shrestha, U.B. and Asselin, H. 2012. Diversity of use and local knowledge of wild edible plant resources in Nepal. Journal of Ethnobiology and Ethnomedicine, 8:16

[37] Xu, J., 2003. Role of indigenous people in biodiversity conservation and utilization in Jinping divide Nature Reserve: an ethnoecological perspective. Chin. J. Ecol. 22 (2), 86-91.

[38] Yeo-Chang, Y. 2009. Use of forest resources, traditional forest-related knowledge and livelihood of forest dependent communities: Case in South Korea. Forest Ecology and Management 257, 2027- 2034. 